\title{
Del comercio electrónico al comercio social: La innovación al alcance de las organizaciones. Estudio para el sector calzado Bucaramanga, Colombia
}

\section{Alba Patricia Guzmán Duque y Carlos Alberto Abreo Villamizar}

\author{
Grupo Estratégico en Investigación Organizacional (Genio) \\ Facultad de Ciencias Económicas, Administrativas y Contables \\ Universidad Autónoma de Bucaramanga
}

Las organizaciones perciben al comercio electrónico como un mecanismo para llegar a sus stakeholders de manera directa, y, de este modo, facilitar el éxito de la transacción desde cualquier punto del mundo (Guzmán, Gil y Carot 2013). El objetivo del artículo es establecer cómo las organizaciones han adoptado esta estrategia para mejorar la eficiencia en la comercialización de sus productos y servicios. Sobre esa base, se evidencia que, para llegar a sus stakeholders, deben orientar sus estrategias hacia la inclusión de la comunidad, a partir de la participación e interacción que se genera con la organización en los medios sociales, entre los cuales Facebook destaca como la red social más utilizada para el e-commerce. Finalmente, se comprueba que el e-commerce ha sobrepasado el simple hecho de comercializar en Internet y ha avanzado hasta el s-commerce o comercio social, un horizonte que incluye a la comunidad y al usuario como partícipes en los procesos relacionados con la comercialización a través de redes sociales.

Palabras clave: comercio electrónico, web social, competitividad, comercio social

\section{From e-commerce to s-commerce: Innovation within the reach of organizations. A study for footwear sector in Bucaramanga, Colombia}

Organizations perceive electronic commerce, as a mechanism to reach their stakeholders directly, facilitating the success of the transaction and from anywhere in the world (Guzman, Gil \& Carot 2013). The objective of this article is to establish how organizations have adopted this strategy to improve efficiency in the marketing of their products and services, showing that to reach their stakeholders they should orient their strategies towards inclusion of the community, from the participation and interaction that is generated with the organization in social internet media, where Facebook is the most used social network for e-commerce. Finally, it is found that e-commerce has surpassed the simple fact of internet marketing, moving up the social commerce or s-commerce, a horizon which includes the user community and the Internet user as a participant in the processes related to marketing through social networks.

Keywords: Telework, social web, competitiveness, social commerce

\section{Do comercio eletronico ao comercio social: A inovação ao alcance das organiçóes}

As organizaçôes percebem ao comercio eletronico, como um mecanismo para chegar ao seus stskeholders de maneira direta, assim facilita o sucesso da negociação e desde qualquer ponto do mundo (Guzmán, Gil \& Carot, 2013). 
O objetivo do artigo é estabelecer cómo as organizaçôes se adaptam esta estrategia para melhorar a eficiencia comercial dos seus productos e serviços, evidenciando que para chegar aos seus stakeholders deben orientar suas estrategias à inclussão da sociedade, partindo da participação e interação que se gera com a organização das redes sociais, onde fecebook é a rede social mais utilizada para o e-comerce. Finalmente, é provado que o e-comerce ultrapassou o simples fato de comercializar na internet, avanzando até o s- comerce ou comercio social, um horizonte que inclui a toda comunidade e ao usuario como participantes nos procesos relacionados com a comercializaçáo por meio das redes sociais.

Palavras chave: comércio eletrônico, web social, competitividade, comércio social.

\section{Introducción}

En la actualidad, el comercio electrónico es una estrategia que utilizan las organizaciones para llegar a más clientes. Sin embargo, en el rubro de calzado de Bucaramanga, en que se ha realizado la investigación, se detecta que este mecanismo aún está lejos de ser extensivo hacia todo el sector. El artículo evidencia la necesidad de mejorar el conocimiento y socializar las ventajas que trae esta estrategia de comercialización, y avanzar hasta el s-commerce como mecanismo que permite que el cliente se sienta partícipe en los procesos de producción y servicios en las empresas, a través de las comunidades que se crean en los medios sociales y que favorecen la inclusión del consumidor como eje principal.

\section{Referente teórico}

\subsection{Web social}

La web social es una plataforma que permite la interacción entre los usuarios de Internet promoviendo la comunicación asertiva. Entre sus ventajas, se encuentra la facilidad para que el usuario acceda directamente a consumir desde cualquier sitio (Grosseck y Holotescu, 2010; Forkosh-Baruch y Hershkovitz, 2011; Guzmán, Gil y Carot, 2013).

Uno de los beneficios que ofrece la web social a las organizaciones es su contribución para la mejora en cuanto a la imagen que promueve a través de la red
(Kierkegaard, 2010). A su vez, facilita la optimización de las estrategias para mantener al consumidor fiel a través del servicio y de su participación en la transacción con su inclusión en el social media o medios sociales de Internet. Además, a través de las relaciones virtuales que se generan, se optimiza el tiempo de los usuarios. Muchas organizaciones prefieren estar al margen de estas tendencias organizacionales, porque están prevenidas por las consecuencias de incluirse, debido a la popularización de los medios sociales como espacios lúdicos, por lo cual desconocen su gran potencial como sistemas de información que les facilita una transacción eficiente (Guzmán y del Moral, 2014).

El social media —o medios sociales, según su traducción al español- es un escenario tecnológico novedoso que fomenta el aprovechamiento de recursos a través de su óptima utilización promoviendo la participación activa de los usuarios (Fuggetta, 2012). Estos crean contenidos, y buscan establecer los lineamientos de los productos o servicios que consumen de manera personalizada (Grosseck y Holotescu, 2010; Forkosh-Baruch y Hershkovitz, 2011). Tal es el caso de las redes sociales, los blogs, las wikis, etc., que reúnen comunidades en torno a un tema en común (Guzmán et al., 2013). De hecho, el ranking de medición del tráfico web Alexa (2015) evidencia dentro de los primeros diez medios sociales más populares en el mundo a Facebook, YouTube, Baidu, Wikipedia y Twitter, lo que corrobora la aseveración anterior. 
Las tecnologías sociales han aportado al advenimiento de diferentes estrategias para las organizaciones que se encaminan a la optimización de recursos y buscan convertirse en novedosos mecanismos para promover procesos más eficientes (Yan, Zheng, Wang, Song y Zhang, 2015), como es el caso del e-commerce.

\subsection{Comercio electrónico o e-commerce}

Las ventajas que ofrece la web social, como la virtualidad y la facilidad de comunicación para acceder desde cualquier lugar, han permitido que, a través de las relaciones, se hagan efectivas y exitosas transacciones en Internet entre las empresas y los consumidores (Kim y Srivastava, 2007). Estos procesos permiten avanzar sobre los medios tradicionales de comercio hasta unos más directos, como el comercio electrónico o e-commerce (Liang y Turban, 2011).

Son diversas las definiciones de e-commerce. Turban, King, Mckay, Marshall y Viehland (2008) indican que es un proceso de compra, venta, transferencia o intercambio de productos, servicios y/o información a través de medios electrónicos. Para Constantinides y Fountain (2008), consiste en la virtualización de los negocios tradicionales, en la que se integra al consumidor como parte activa de las estrategias basadas en sus experiencias de compra y la solución a sus expectativas para generar una transacción exitosa. De otro lado, Yapar, Bayrakdar y Yapar (2015) aseguran que el comercio electrónico permite a las empresas vender sus productos y servicios con diferentes métodos en todo el mundo, $y$ a los consumidores les permite acceder a ellos con facilidad. Finalmente, Liang y Turban (2011) afirman que es un espacio en que los consumidores colaboran en línea; obtienen asesoramiento de personas de confianza; descubren productos y servicios; y, luego, los consumen.

El comercio electrónico es un sitio en el cual, a partir de la colaboración en línea entre los actores, organiza- ciones y consumidores, se cierra un negocio utilizando las tecnologías sociales, la interacción entre las comunidades y la transacción (Liang y Turban, 2011). En ese sentido, el e-commerce gira en un entorno social, puesto que se desarrolla a través de la participación de los usuarios y la generación de la conversación en línea que aportan al cierre de un proceso comercial (Huang y Benyoucef, 2013). Según Liang y Turban (2011), existen tres atributos del e-commerce: las tecnologías de medios sociales, las interacciones de la comunidad y las actividades comerciales. Dichos atributos propician un espacio respaldado por la comunidad a través de la participación y la interacción, en el que fomentan la transacción a través de su relación. La tabla 1 evidencia los beneficios del e-commerce, como espacio para generar diversidad de estrategias basadas en la utilización de la tecnología tanto física como social para hacer más eficientes los procesos, y la inversión en costos y gastos.

\section{Tabla 1. Beneficios del $\boldsymbol{e}$-commerce}

\begin{tabular}{|l|l|}
\hline \multicolumn{1}{|c|}{ Beneficios del comercio electrónico } & \multicolumn{1}{|c|}{ Autores } \\
\hline $\begin{array}{l}\text { Aporta a la reducción de costos en los proce- } \\
\text { sos, promueve la apertura de nuevos mercados, } \\
\text { mejora la atención al cliente y capta a nuevos. }\end{array}$ & $\begin{array}{l}\text { Romero y } \\
\text { Mauricio } \\
(2012)\end{array}$ \\
\hline $\begin{array}{l}\text { Permite a los clientes el acceso directo sin con- } \\
\text { tacto presencial a las compras, con lo cual facilita } \\
\text { la transacción desde cualquier sitio del mundo. }\end{array}$ & $\begin{array}{l}\text { Guzmán, Gil y } \\
\text { Carot (2013) }\end{array}$ \\
\hline $\begin{array}{l}\text { Al eliminar a los intermediarios, proporciona } \\
\text { la optimización de los procesos debido a la } \\
\text { personalización de los deseos del consumidor. }\end{array}$ & $\begin{array}{l}\text { Lopes y Roy } \\
(2015)\end{array}$ \\
\hline $\begin{array}{l}\text { Su adopción en las economías en desarrollo } \\
\text { les permite a las pequeńas y medianas empre- } \\
\text { sas sobrevivir en la era de la información. }\end{array}$ & $\begin{array}{l}\text { Rahayu y Day } \\
(2015)\end{array}$ \\
\hline $\begin{array}{l}\text { Facilita la venta de bienes y servicios a través } \\
\text { de diferentes métodos alrededor del mundo. }\end{array}$ & $\begin{array}{l}\text { Yapar, Bayrakdar } \\
\text { y Yapar (2015) }\end{array}$ \\
\hline $\begin{array}{l}\text { Otorga altos niveles de reputación en el } \\
\text { mercado, a partir de lo cual se reduce la } \\
\text { información asimétrica y se incrementa su } \\
\text { aceptación en el mercado. }\end{array}$ & $\begin{array}{l}\text { Xiao y Dong } \\
\text { (2015) }\end{array}$ \\
\hline
\end{tabular}

Contabilidad y Negocios (12) 24, 2017 / ISSN 1992-1896 
Sin embargo, el consumidor requiere sentirse tranquilo en el sitio donde realizará la transacción, lo cual obliga a las organizaciones a ofrecerles seguridad y confiabilidad (Hart y Saunders, 1997; Huang y Benyoucef, 2013), que aseguren fiabilidad en la transacción para garantizar su éxito (Ratnasingham, 1998). En ese contexto, precisamente, las oportunidades que ofrece el e-commerce a las organizaciones generalmente son limitadas a causa de diversos factores, como se observa en la tabla 2.

\section{Tabla 2. Causas que limitan la implementación} del $e$-commerce

\begin{tabular}{|l|l|}
\hline $\begin{array}{l}\text { Factores que limitan la implementación } \\
\text { del } \text {-commerce }\end{array}$ & \multicolumn{1}{|c|}{ Autores } \\
\hline $\begin{array}{l}\text { Hay una baja adopción de las TIC en las } \\
\text { empresas, debido a la desconfianza alre- } \\
\text { dedor de la transacción y los costos de } \\
\text { implementación. }\end{array}$ & $\begin{array}{l}\text { Romero y Mauricio } \\
\text { (2012), y Nilashi, } \\
\text { Ibrahim, Reza } \\
\text { Mirabi, Ebrahimi y } \\
\text { Zare (2015) }\end{array}$ \\
\hline $\begin{array}{l}\text { Los costos en su operación pueden } \\
\text { incrementarse, debido a las diferencias } \\
\text { lingüísticas, culturales, institucionales y } \\
\text { comerciales. }\end{array}$ & $\begin{array}{l}\text { Gómez-Herrera, } \\
\text { Martens y Turlea }\end{array}$ \\
\hline $\begin{array}{l}\text { Existen limitaciones técnicas y no téc- } \\
\text { nicas relacionadas con la seguridad y la } \\
\text { infraestructura que las provee, y de la cual } \\
\text { la organización carece en ocasiones. }\end{array}$ & $\begin{array}{l}\text { Savrul, Incekara y } \\
\text { Sener (2014) }\end{array}$ \\
\hline $\begin{array}{l}\text { El proceso de implementación en las } \\
\text { pymes de los países en desarrollo ha } \\
\text { sido lento por las condiciones sociales, } \\
\text { económicas, tecnológicas y políticas des- } \\
\text { favorables. }\end{array}$ & $\begin{array}{l}\text { Kurnia, Choudrie, } \\
\text { Alzougool (2015) }\end{array}$ \\
\hline $\begin{array}{l}\text { La incertidumbre tributaria y la doble } \\
\text { imposición de algunas modalidades de } \\
\text { pago generan renuencia a su implemen- } \\
\text { tación. }\end{array}$ & $\begin{array}{l}\text { Yapar, Bayrakdar y } \\
\text { Yapar (2015) }\end{array}$ \\
\hline
\end{tabular}

Las respuestas de los autores evidencian que, si bien el e-commerce ofrece beneficios o ventajas para los empresarios, se presentan inconvenientes, como la falta de confianza y el conocimiento sobre su desarrollo e implementación, debido al desconocimiento sobre esta estrategia de comercialización. Pese a ello, existen diferentes sectores que han visto la importancia del comercio electrónico (Aguilar-Jiménez, Luzardo Briceño, Jaimes Carrillo y Rojas Hernández, 2013). Tal es el caso del sector joyero de Bucaramanga, cuyos empresarios han indicado que es un mecanismo para exponer sus productos y comunicarse con los clientes, pero no para cerrar transacciones. Cabe anotar que esto lo indica el 5\% de los empresarios.

Finalmente, es importante resaltar que la adopción del e-commerce en las organizaciones debe ser implementada de acuerdo con la naturaleza de la actividad y a su entorno, como lo indican Cui y Pan (2015). Estos autores revelan que su implementación obedece a tres aspectos para alcanzar el éxito en la transacción: el desarrollo de capacidades para lograr el éxito considerando el entorno y los recursos, la gestión de la estructura y la estrategia para explotar las capacidades existentes, y la gestión de los recursos para generar nuevos recursos y capacidades dinámicas.

\subsection{Comercio social o s-commerce}

La comercialización de los productos no es una práctica nueva, pues siempre ha existido. La diferencia actual reside en la manera en que se desarrolla este proceso. De hecho, el e-commerce continúa su desarrollo como estrategia con la utilización de los medios sociales para convertirse en el s-commerce (Wu, Shen y Chang, 2015). En ese marco, las herramientas sociales de Internet —blogs, redes sociales, wikis, etc.— aportan al desarrollo del comercio electrónico orientándose hacia el comercio social, en la medida que consideran al usuario, la interacción con la comunidad y la transacción (Huang y Benyoucef 2013; Bai, Yao y Dou, 2015). De este modo, permiten que se genere una comunicación efectiva entre dichas comunidades; 
a través de estas conversaciones online, se promueve la personalización de productos y servicios para el cliente y su comunidad (Boyd y Ellison, 2007; Alloway y Alloway, 2012; Linvill, McGee y Hicks, 2012). Según Hajli y Sims (2015), el s-commerce se desarrolló por la creciente popularidad de las redes sociales y su integración en plataformas de e-commerce. Esto ha proporcionado valores como la cocreación de contenido, que se desarrolla por la colaboración entre consumidores y empresas, lo cual agrega valor al proceso, y al producto o servicio.

La revisión de la literatura ofrece diversas aproximaciones hacia una definición del s-commerce. Para Wu et al. (2015), es un medio en línea que combina tecnologías de la web social y la satisfacción que obtiene el consumidor al realizar una transacción referida por una comunidad virtual. Dentro de estas herramientas, se encuentran las redes sociales, los blogs, las comunidades en línea, los foros, las opiniones y las recomendaciones (Hajli y Sims, 2015). Zhang, Lu, Gupta y Zhao (2014) añaden a esta lista los microblogs, las wikis, las plataformas de comercio electrónico y otros sitios de contenido compartido, como YouTube y Flickr. Estos influyen en la comunidad que gira a su alrededor $y$, por tanto, este aspecto incide en el éxito de la transacción (Bai, Yao y Dou, 2015; Huang y Benyoucef, 2015). De hecho, Yan et al. (2015) aseguran que este es un nuevo y prometedor paradigma del comercio electrónico, que se caracteriza por su carácter dinámico a través de los mecanismos de reputación. Así mismo, Qin y Kong (2015) destacan la utilidad de las recomendaciones realizadas por otros usuarios para el desarrollo de transacciones en plataformas de comercio social. De otro lado, se aprecia que, a través del comercio social, las organizaciones interactúan directamente con los consumidores para cerrar la transacción, lo cual fomenta la lealtad de marca.

Es importante indicar que existen diferencias entre el e-commerce y el s-commerce, que van más allá de la evolución del primero hacia el segundo, como se muestra en la siguiente tabla.

\section{Tabla 3. Diferencias entre el e-commerce y el s-commerce}

\begin{tabular}{|l|l|l|l|}
\hline & $\begin{array}{l}\text { e-commerce } \\
\text { o comercio } \\
\text { electrónico }\end{array}$ & $\begin{array}{l}\text { s-commerce o } \\
\text { comercio social }\end{array}$ & \multicolumn{1}{|c|}{ Autores } \\
\hline $\begin{array}{l}\text { Forma de } \\
\text { comunicación }\end{array}$ & $\begin{array}{l}\text { Atención } \\
\text { personalizada }\end{array}$ & $\begin{array}{l}\text { Interacción con } \\
\text { los clientes }\end{array}$ & $\begin{array}{l}\text { Bai, Yao y } \\
\text { Dou (2015) }\end{array}$ \\
\hline $\begin{array}{l}\text { Atención al } \\
\text { cliente }\end{array}$ & $\begin{array}{l}\text { Personalizada } \\
\text { individual }\end{array}$ & $\begin{array}{l}\text { Personalizada } \\
\text { considerando la } \\
\text { comunidad }\end{array}$ & $\begin{array}{l}\text { Huang y } \\
\text { Benyoucef } \\
(2013)\end{array}$ \\
\hline Relaciones & Estáticas & Dinamizadoras & $\begin{array}{l}\text { Yan, Zheng, } \\
\text { Wang, Song } \\
\text { y Zhang } \\
(2015)\end{array}$ \\
\hline Información & Asimétrica & $\begin{array}{l}\text { Cocreación de } \\
\text { contenidos a } \\
\text { través de la cola- } \\
\text { boración entre } \\
\text { consumidores y } \\
\text { empresas }\end{array}$ & $\begin{array}{l}\text { Hajli y Sims } \\
(2015)\end{array}$ \\
\hline $\begin{array}{l}\text { Utilización } \\
\text { de los medios } \\
\text { sociales }\end{array}$ & $\begin{array}{l}\text { Para comuni- } \\
\text { carse }\end{array}$ & $\begin{array}{l}\text { Para cerrar la } \\
\text { transacción }\end{array}$ & $\begin{array}{l}\text { Wu, Shen } \\
\text { y Chang } \\
(2015)\end{array}$ \\
\hline
\end{tabular}

Entre las características necesarias para generar un ambiente que promueva el comercio social, se encuentran el individuo, la conversación, la comunidad y el comercio (Huang y Benyoucef, 2013).

\section{Metodología}

La investigación se realizó en el sector del calzado en la ciudad de Bucaramanga, compuesto por 1424 empresas de calzado — 69 sociedades y 1355 empresas 
son de personas naturales - dedicadas en mayor parte a la fabricación de calzado de dama, entre las cuales el 93\% son microempresas; y el 7\%, pymes. La mayoría de empresas producen su propia marca y solo un 1,7\% maquila. Además, el 43,3\% exporta marca propia; y el 13,3\%, otra marca (Compite360, 2014).

La metodología utilizada en este artículo es cuantitativa y la muestra se calculó sobre la base de los parámetros del muestreo aleatorio simple (Sampieri, collado, Bautista y de la Cruz, 1998), considerando un $95 \%$ de confiabilidad y un error máximo del $9 \%$. El resultado fue de 110 encuestas a realizar, de las cuales se obtuvo respuesta de 113 empresas de calzado de Bucaramanga. La recolección de la información se realizó en abril de 2015 para investigar la utilidad que perciben los empresarios del sector con la utilización del e-commerce como mecanismo estratégico para llegar a sus stakeholders y su consideración de las herramientas de los medios sociales como mecanismos para avanzar en el proceso hacia el s-commerce. El instrumento utilizado fue una encuesta virtual y medida bajo la escala de Likert ( 1 a 5 , en que 1 corresponde a "Totalmente en desacuerdo"; y 5, a "Totalmente de acuerdo"). La composición del instrumento se basó en cuatro apartados: (i) elementos descriptivos de la organización, (ii) utilización del e-commerce y de los medios sociales, (iii) adopción del teletrabajo, y (iv) competencias del teletrabajador. La viabilidad del instrumento fue comprobada a través del alfa de Cronbach que arrojó como resultado el 0,83.

En cuanto a las técnicas estadísticas que se utilizaron para el análisis de datos, además de las descriptivas, se optó por la Tau-b de Kendall para medir las relaciones entre las variables categóricas (Hair, Prentice, Cano y Suárez 2007) con el paquete estadístico SPSS (v.18).

\section{Hallazgos y su interpretación}

\subsection{Descripción de la muestra}

El 52,2\% de las empresas comercializadoras de calzado de la ciudad de Bucaramanga tienen una antigüedad de 3 a 5 años; el 26,5\% tienen más de 5 años; el $18,6 \%$, entre 1 y 3 años; y solo el $2,7 \%$ fueron creadas hace menos de un año. Por otro lado, el $44,2 \%$ de las empresas cuentan con 1 a 5 empleados; el 31,0\%, con 5 a 10 empleados; y el 24,7\% cuenta con más de 10 empleados. Ello evidencia que las empresas comercializadoras de calzado de la ciudad de Bucaramanga tienen un tamaño pequeño, lo cual puede impedir un mayor desarrollo del negocio en el ámbito internacional.

\subsection{Acercamiento al e-commerce en el sector calzado}

El 30,1\% de los empresarios afirma que en las empresas comercializadoras de calzado se utiliza el e-commerce, mientras que el $20,4 \%$ no lo hace y un $49,5 \%$ indica que lo hace poco. Este aspecto es preocupante si se considera que, al utilizar esta estrategia de comercialización, lo más coherente sería que la mitad de los empresarios no lo utilizase; sin embargo, esa mitad de los empresarios que indica que sí lo hace pero muy poco demuestra el desconocimiento sobre el tema.

En cuanto a las consideraciones de los empresarios referentes al e-commerce, el $84,1 \%$ señala que es importante esta estrategia, porque permite incrementar los lazos comerciales; el 84,0\% afirma que permite abrir nuevos mercados; el 76,1\% lo hace para aumentar el número de clientes; el $76.1 \%$ asegura que es una forma de comercializar innovadora; y el 67,3\%, que sirve para la negociación. Por su parte, el 61,1\% asegura que promueve la contratación de empleados; el 54,9\%, que mejora el servicio al cliente; el 47,8\%, que ofrece tanto ventajas como desventajas; el 39,0\%, 
que disminuye los gastos; y el 35,9\%, que reduce los costos. Si se lee entre líneas, se observa que son más las ventajas que las desventajas que ofrece este sistema de comercialización para los empresarios. Pese a que ello, no lo reconocen abiertamente. Esto se podría explicar por la desconfianza que se genera alrededor de las transacciones en Internet debido al desconocimiento de medios seguros para cerrarlas.

El gráfico 1 muestra los objetivos del e-commerce. Al preguntarles sobre la finalidad de esta herramienta, el $74,4 \%$ de los empresarios indicó que el principal fin de esta estrategia es comercializar la marca de la empresa, seguido por el 54,9\%, que señaló que se orienta a actualizar el destino de ventas de la organización. Para el 43,4\%, el objetivo es ofrecer los productos a través de la web de la organización, mientras que, para el 38,9\%, incrementar el número de clientes de la compañía. Finalmente, el 22,2\% sostiene que la finalidad es ofrecer portales para dar a conocer el producto. Se destaca en este apartado que el $58,4 \%$ de ellos afirman que los portales no son tan importantes como un objetivo a desarrollar con el e-commerce.
Los análisis revelan que las empresas comercializadoras de calzado conocen los mecanismos de e-commerce, pero no lo implementan, según lo afirma el 43,4\%. Además, el 40,7\% tiene poco conocimiento sobre el funcionamiento de esta estrategia; el 33,6\% asegura que el personal conoce de tecnologías, pero no está formado para desarrollar esta tarea; y el $26,7 \%$ no sabe cómo implementar este proceso de comercialización. A ello se suma que el $57,5 \%$ de los empresarios saben cómo implementar el e-commerce; no obstante, aún no lo ha implementado.

\subsection{El comercio social: la evidencia de la innovación}

En cuanto a la utilización de los medios sociales y su acercamiento del e-commerce al s-commerce, se detecta que el $64,1 \%$ utiliza redes sociales. Entre ellas, Facebook es la más popular como canal de venta online. Más bien, se desconocen los canales especializados (gráfico 2), como Mercado Libre (11,5\%), Amazon (4,4\%), Linio (3,5\%), OLX (3,5\%). Tan solo el $1,8 \%$ indican haber utilizado Ebay y Taobao.

Gráfico 1. Distribución porcentual de los objetivos del e-commerce

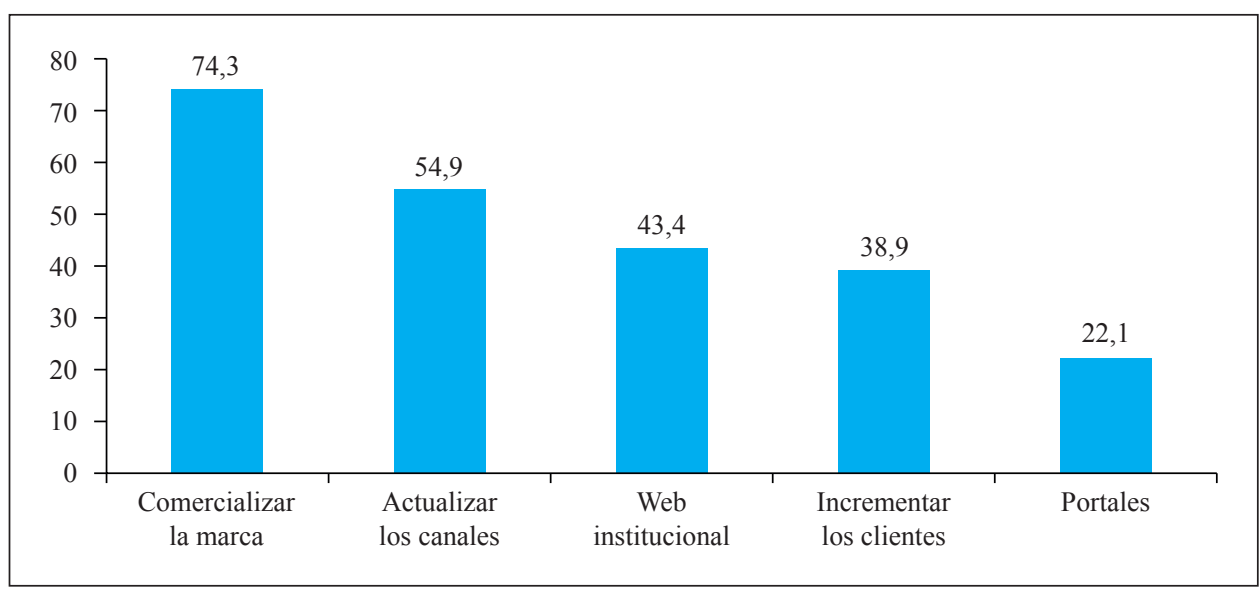


Gráfico 2. Distribución porcentual de la utilización de los canales de e-commerce

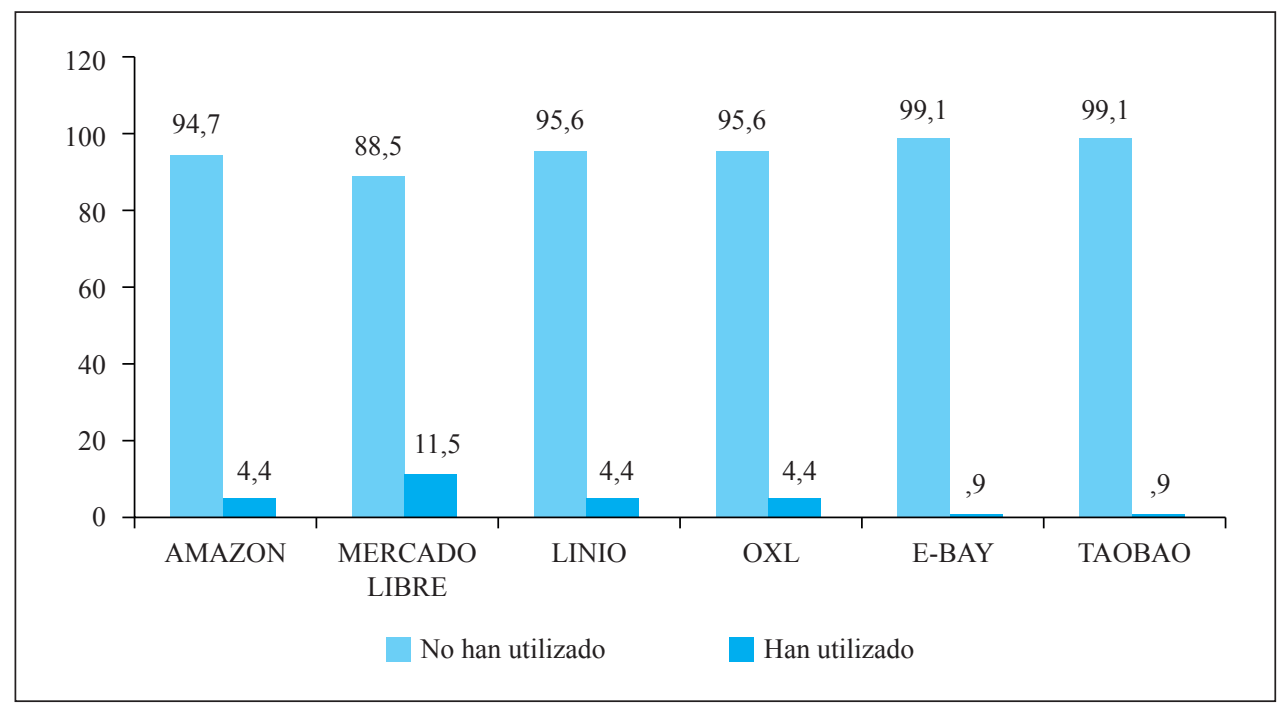

Es importante destacar que la utilización de los canales de e-commerce no son considerados por los empresarios del calzado en Bucaramanga. Pese a que reconocen que existen, el desconocimiento del medio de transacción en Internet implica que estos eviten su utilización, por lo cual prefieren hacerlo a través de las redes sociales. Aun cuando la seguridad de estas en la transacción es menor, se ven respaldados por una comunidad que ofrece confianza para realizar la negociación.

En cuanto a los medios sociales en Internet, se confirma que Facebook es la red más utilizada, según lo indica el 60,3\%; seguido por Instagram, con 32,7\%; la publicidad a través de los blogs (privados el 22,1\% y públicos el 13,3\%); y Twitter, con el 9,7\%. Se debe anotar que casi no se utilizan otros medios conocidos, como YouTube, LinkedIn, Wikipedia y Baidu (gráfico 3). A partir de ello, se observa que Facebook sigue siendo el medio social más popular. Esto debe conducir al empresario hacia la utilización de estas herramientas sociales para aprovechar el nicho de mercado que tiene en la red social, que le permite ir directamente al usuario considerando su comunidad.

$\mathrm{Al}$ realizar las correlaciones bivariadas entre las variables, se detecta que, en las razones para utilizar el e-commerce, hay correlaciones positivas y significativas entre las negociaciones y el mayor número de clientes $(\mathrm{W}=, 743)$, y el servicio al cliente $(\mathrm{W}=, 648)$, y la promoción del trabajo $(\mathrm{W}=, 687)$, además de favorecer la comercialización (W = ,647). Esto indica que la negociación es el principal motor que mueve al s-commerce y que, efectivamente, se busca siempre incrementar el número de clientes a través del servicio como mecanismo de promoción.

Por otro lado, también hay correlaciones positivas y significativas entre las empresas que afirman que es posible aumentar el número de clientes a partir de la comercialización (W = ,727); además, disminuye los costos y los gastos ( $\mathrm{W}=, 867)$, y permite acceder a nuevos mercados, porque favorece la comercialización (W = ,785). Esto muestra que las empresas que 
Gráfico 3. Distribución porcentual de la utilización de los medios sociales

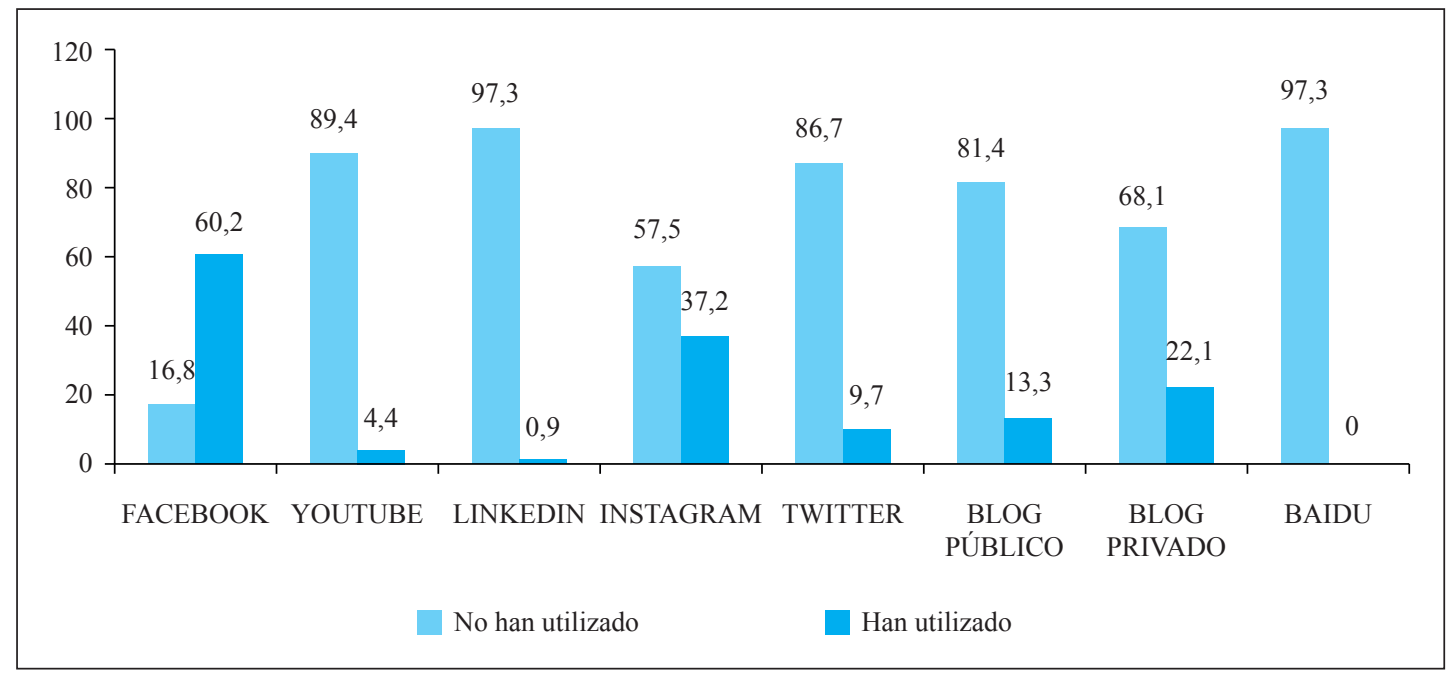

utilizan este mecanismo - y que indican ser no más de la mitad - realmente ven la potencialidad de esta estrategia innovadora como elemento dinamizador del mercado y de su mercado natural.

Entre los objetivos que se publican a través de la web y la importancia de alcanzar a los consumidores con la negociación, se encuentra una correlación positiva y significativa (W = ,634). Ello muestra que, efectivamente, las publicaciones que se realizan en Internet son consideradas como mecanismos para llegar a los clientes. Sin embargo, sí preocupa que, a pesar de que se reconoce la importancia de este medio de comunicación, no es considerado como un mecanismo que facilite el comercio social.

Entre la estrategia del comercio en redes sociales con el grado de utilización del e-commerce en la empresa (W = 596), se indica que el s-commerce llegó para quedarse, pues a través de las comunidades que se crean en las redes sociales se respaldan las transacciones realizadas entre empresas y consumidores, y se desarrolla un vínculo importante que posibilita el incremento de las ventas, la disminución de los costos y el acceso a nuevos mercados.

Entre los problemas para la implementación del e-commerce se destacan las apreciaciones sobre el objetivo de comercializar la marca $(\mathrm{W}=, 612)$, con el conocimiento sobre el e-commerce (W = ,653), la capacitación sobre e-commerce del personal (W = ,616). Esto da cuenta de que es evidente la apropiación del conocimiento sobre estas estrategias tecnológicas para mejorar la comercialización de los productos o servicios de una organización.

Finalmente, al revisar la correlación entre la comercialización de productos a través de los medios sociales, se detecta que los empresarios realizan las ventas en medios sociales y en Facebook $(\mathrm{W}=, 689)$, con lo cual se obtiene una correlación significativa y positiva. De este modo, se evidencia que reconocen al s-commerce como una estrategia innovadora en su proceso de comercialización. 


\section{Conclusiones}

Las empresas comercializadoras de calzado de la ciudad de Bucaramanga reflejan interés por la adopción del comercio electrónico como mecanismo para mejorar sus indicadores; no obstante, aún temen hacer inversiones al respecto por el desconocimiento de su implementación y la desconfianza que se genera alrededor de las negociaciones en la red.

La interacción y la comunicación que se genera a través del uso de los medios sociales de Internet permiten que el usuario de la red se convierta en actor principal, en la medida que puede participar con voz y voto a la hora de comercializar un producto o servicio de manera eficiente para consumir en comunidad.

Es evidente que el s-commerce es una tendencia que las organizaciones requieren adoptar para establecer sus estrategias a corto, mediano y largo plazo, puesto que esto les ofrece oportunidades innovadoras en la comercialización, las cuales ya están siendo aprovechadas por pequeños comerciantes que han visto sus potencialidades.

\section{Referencias}

Aguilar-Jiménez, Alba Soraya, Marianela Luzardo Briceño, Ludym Jaimes Carillo y Guillermo Andrés Rojas Hernández (2013). Comercio electrónico en empresas del sector de comercialización de joyas de Bucaramanga Colombia. Ponencia presentada en el $V$ Congreso Iberoamericano Socote - Soporte del Conocimiento con la Tecnología. Universidad de San Martín de Porres. Lima, 15 y 16 de octubre.

Alexa (2015). The top sites on the web. Alexa. Recuperado el 15 de noviembre de 2017 de http://www.alexa.com/ topsites

Alloway, Tracy Packiam y Ross Geoffrey Alloway (2012). The impact of engagement with social networking sites (SNSs) on cognitive skills. Computers in Human Behavior, 28(5), 1748-1754. https://doi. org/10.1016/j.chb.2012.04.015

Bai, Yai, Zhong Yao y Yi-Fan Dou (2015). Effect of social commerce factors on user purchase behavior: An empirical investigation from renren.com. International Journal of Information Management, 35(5), 538-550. https://doi.org/10.1016/j.ijinfomgt.2015.04.011

Boyd, Danah y Nicole Ellison (2007). Sitios de redes sociales: Definición, historia y conocimiento. Journal of Computer-Mediated Communication, 12(1).

Compite360. Información Empresarial De Colombia (2014). Sistema de consulta información comercial, financiera, jurídica y económica de empresas en Colombia legalmente constituidas en las diferentes Cámaras de Comercio del país.

Constantinides, Efthymios y Stefan Fountain (2008). Web 2.0: Conceptual foundations and marketing issues. Journal of Direct, Data and Digital Marketing Practice, 9(3), 231-244. https://doi.org/10.1057/palgrave. dddmp.4350098

Cui, Miao y Shan Pan (2015). Developing focal capabilities fore-commerceadoption: Aresourceorchestration perspective. Information \& Management, 52(2), 200-209. https://doi.org/10.1016/j.im.2014.08.006

Forkosh-Baruch, Alona y Arnon Hershkovitz (2011). The use of social networks by higher-education institutes in Israel. Proceedings of the Chair Conference on Instructional Technologies Research), 14-20.

Fuggetta, Alfonso (2012). 3+ 1 Challenges for the Future of Universities. Journal of Systems and Software, 85(10), 2417-2424. https://doi.org/10.1016/j.jss. 2012.05 .062

Gómez-Herrera, Estrella, Bertin Martens y Geomina Turlea (2014). The drivers and impediments for cross-border e-commerce in the EU. Information Economics and Policy, 28), 83-96. https://doi.org/10.1016/j.infoecopol.2014.05.002 
Grosseck, Gabriela y Carmen Holotescu (2010). Microblogging multimedia-based teaching methods best practices with Cirip. Eu. Procedia-Social and Behavioral Sciences, 2(2), 2151-2155. https://doi.org/10.1016/j. sbspro.2010.03.297

Guzmán, Alba Patricia, Hermenegildo Gil y Jose Miguel Carot (2013). Factores criticos de éxito en el uso de las redes sociales en el ámbito universitario: Aplicación a Twitter. Valencia: Editorial Universitat Politécnica de Valéncia.

Guzmán, Alba Patricia y Maria Esther Del Moral (2014). Tendencias de uso de YouTube: Optimizando la comunicación estratégica de las universidades iberoamericanas. Observatorio $\left(O B S^{*}\right), 8(1), 69-94$.

Hair, Joseph, Rolph Prentice, Diego Cano y Monica Gómez Suárez (2007). Análisis multivariante. Quinta edición. Madrid: Prentice Hall.

Hajli, Nick y Julian Sims (2015). Social commerce: The transfer of power from sellers to buyers. Technological Forecasting and Social Change, 94), 350-358. https:// doi.org/10.1016/j.techfore.2015.01.012

Hart, Paul y Carol Saunders (1997). Power and trust: Critical factors in the adoption and use of electronic data interchange. Organization Science, 8(1), 23-42. https://doi.org/10.1287/orsc.8.1.23

Huang, Zhao y Morad Benyoucef (2013). From e-commerce to social commerce: A close look at design features. Electronic Commerce Research and Applications, 12(4), 246-259. https://doi.org/10.1016/j. elerap.2012.12.003

Huang, Zhao y Morad Benyoucef (2015). User preferences of social features on social commerce websites: An empirical study. Technological Forecasting and Social Change, 95, 57-72. https://doi.org/10.1016/j.techfore.2014.03.005

Kierkegaard, Sylvia (2010). Twitter thou doeth?”. Computer Law \& Security Review, 26(6), 577-594. https://doi. org/10.1016/j.clsr.2010.09.002
Kim, Young y Jaideep Srivastava (2007). Impact of social influence in e-commerce decision making. Proceedings of the ninth international conference on Electronic commerce (pp. 293-302) Nueva York: ACM. https:// doi.org/10.1145/1282100.1282157

Kurnia, Sherah, Jyoti Choudrie, Rahim Md Mahbubur y Basil Alzougool (2015). E-commerce technology adoption: A Malaysian grocery SME retail sector study. Journal of Business Research, 68(9), 1906-1918. https://doi.org/10.1016/j.jbusres.2014.12.010

Liang, Ting-Peng y Efraim Turban (2011). Introduction to the special issue social commerce: a research framework for social commerce. International Journal of Electronic Commerce, 16(2), 5-14. https://doi.org/10.2753/ jec1086-4415160201

Linvill, Darren, Sara McGee y Laura Hicks (2012). Colleges' and universities' use of Twitter: A content analysis. Public Relations Review, 38(4), 636-638. https://doi.org/10.1016/j.pubrev.2012.05.010

Lopes, Prajyoti y Bidisha Roy (2015). Dynamic recommendation system using web usage mining for e-commerce users. Procedia Computer Science, 45, 60-69. https:// doi.org/10.1016/j.procs.2015.03.086

Nilashi, Mehrbakhsh, Othman Ibrahim, Vahid Reza Mirabi, Leili Ebrahimi y Mojtaba Zare (2015). The role of security, design and content factors on customer trust in mobile commerce. Journal of Retailing and Consumer Services, 26, 57-69. https://doi.org/10.1016/j. jretconser.2015.05.002

Qin, Li y Sue Kong (2015). Perceived helpfulness, perceived trustworthiness, and their impact upon social commerce users" intention to seek shopping recommendations". Journal of Internet Commerce, 14(4), 492-508. https:// doi.org/10.1080/15332861.2015.1103634

Rahayu, Rita y John Day (2015). Determinant factors of e-commerce adoption by smes in developing country: Evidence from Indonesia. World Conference on 
Technology, Innovation and Entrepreneurship, 195, 142150. https://doi.org/10.1016/j.sbspro.2015.06.423

Ratnasingham, Pauline (1998). The importance of trust in electronic commerce. Internet Research: Electronic Networking Applications and Policy, 8(4), 313-321.

Romero, Pablo y David Mauricio (2012). Revisión de modelos de adopción de e-commerce para pymes de países en desarrollo. Revista de Investigación de Sistemas e Informática, 9(1), 69-90.

Sampieri, Roberto, Carlos Fernando Collado, Maria del Pilar Bautista Lucio y Maria de la Cruz Casas Pérez (1998). Metodología de la investigación, 1. México D.F.: McGraw-Hill.

Savrul, Mesut, Ahmet Incekara y Sefer Sener (2014). The potential of e-commerce for SMEs in a globalizing business environment. Procedia-Social and Behavioral Sciences, 150, 35-45. https://doi.org/10.1016/j.sbspro.2014.09.005

Turban, Efraim, David King, Judy Mckay, Peter Marshall, Jae Lee y Dennis Viehland (2008). Electronic commerce: A managerial perspective. Quinta edición. New Jersey: Prentice Hall.

Wu, Yen-Chun Jim, Ju-Peng Shen y Chan-Lan Chang (2015). Electronic service quality of Facebook social commerce and collaborative learning. Computing for human learning, behaviour and collaboration in the social and mobile networks era, 51, Part B), 1395-1402. https://doi.org/10.1016/j.chb.2014.10.001
Xiao, Shengsheng y Ming Dong (2015). Hidden semiMarkov model-based reputation management system for online to offline $(\mathrm{O} 2 \mathrm{O})$ e-commerce markets. Decision Support Systems, 77, 87-99. https://doi. org/10.1016/j.dss.2015.05.013

Yan, Su-Rong, Xiao-Lin Zheng, Yang Wang, William Wei Song y Wen-Yu Zhang (2015). A graph-based comprehensive reputation model: Exploiting the social context of opinions to enhance trust in social commerce. Security, Privacy and Trust in Network-Based Big Data, 318, 51-72. https://doi.org/10.1016/j. ins.2014.09.036

Yapar, Burcu Kuzucu, Seda Bayrakdar y Mustafa Yapar (2015). The role of taxation problems on the development of e-commerce. Procedia - Social and Behavioral Sciences, 195, 642-648. https://doi.org/10.1016/j.sbspro.2015.06.145

Zhang, Hong, Yaobin Lu, Sumeet Gupta y Ling Zhao (2014). What motivates customers to participate in social commerce? The impact of technological environments and virtual customer experiences. Information \& Management, 51(8), 1017-1030. https://doi. org/10.1016/j.im.2014.07.005

Recibido: 23/04/2017 Aceptado: 24/10/2017 albapatrig@gmail.com carlosabreo@gmail.com 